Research Article

\title{
Cross-Sectional Relationship between Carotid-Femoral Pulse Wave Velocity and Biomarkers in Vascular-Related Diseases
}

\author{
Jinbo Liu $\left(\mathbb{D},{ }^{1}\right.$ Kuanting Wang, ${ }^{2}$ Huan Liu $\mathbb{D},{ }^{1}$ Hongwei Zhao, ${ }^{1}$ Wei Huang, ${ }^{1}$ Na Zhao, \\ Lihong Li, ${ }^{1}$ and Hongyu Wang $\mathbb{D D}^{1}$ \\ ${ }^{1}$ Department of Vascular Medicine, Peking University Shougang Hospital, Beijing 100144, China \\ ${ }^{2}$ Department of Rheumatology and Immunology, Peking University Shougang Hospital, Beijing 100144, China
}

Correspondence should be addressed to Hongyu Wang; hongyuwang@188.com

Received 24 August 2019; Accepted 12 May 2020; Published 25 May 2020

Academic Editor: Tomohiro Katsuya

Copyright (c) 2020 Jinbo Liu et al. This is an open access article distributed under the Creative Commons Attribution License, which permits unrestricted use, distribution, and reproduction in any medium, provided the original work is properly cited.

\begin{abstract}
Objectives. The present study was done to investigate the relationship between carotid-femoral pulse wave velocity (CFPWV) and biomarkers such as homocysteine (Hcy), N-terminal pro-brain natriuretic peptide (NT-proBNP), and urine albumin (microalbumin) (UAE) in vascular-related diseases. Methods. 656 subjects were enrolled into our study. There were 377 patients with hypertension, 231 with coronary heart disease, 154 with diabetes mellitus, and 186 healthy subjects. They were divided into four groups according to the number of suffered diseases: group 1 had only one of three diseases, group 2 had two, and group 3 had all of three diseases. CFPWV was measured by Complior apparatus. Results. CFPWV was significantly higher in group 3 than in the healthy group, group 1 , and group $2(12.71 \pm 2.38$ vs $10.11 \pm 2.28,10.70 \pm 2.12$, and $11.92 \pm 2.55$, all $p<0.05)$. The level of Hcy was significantly higher in group 1, group 2, and group 3 than in healthy subjects, respectively. Levels of Log NT-proBNP and Log UAE were significantly higher in group 3 than in group 1 ( $2.27 \pm 0.4$ vs $2.10 \pm 0.4,1.00 \pm 0.65$ vs $0.68 \pm 0.56$, both $p<0.05)$. Positive correlation between CFWV and Hcy, Log UAE, and Log NT-proBNP was found in the entire study group $(r=0.109,0.196$, and 0.119 , all $p<0.05)$. Multivariate analysis showed that pulse pressure, age, fasting plasma glucose, diastolic blood pressure, body mass index, and Log UAE were independent associating factors of CFPWV in all subjects $(\beta=0.334, p<0.001 ; \beta=0.333$, $p<0.001 ; \beta=0.126, p=0.004 ; \beta=0.137, p=0.003 ; \beta=-0.142, p=0.002$; and $\beta=0.098, p=0.031)$. Conclusions. CFPWV was significantly higher in subjects with hypertension, $\mathrm{CHD}$, and DM. There was correlation between CFPWV and biomarkers such as NT-proBNP, Hcy, and urine albumin (microalbumin).
\end{abstract}

\section{Introduction}

Coronary heart disease (CHD), hypertension, and diabetes mellitus (DM) are the most serious vascular-related diseases threatening the human's life. Arteriosclerosis is the basic pathophysiological change of these diseases, and it can be measured by arterial stiffness. A recent study showed that arterial stiffness is a strong predictor of future cardiovascular events and all-cause mortality [1]. Carotid-femoral pulse wave velocity (CFPWV) is considered as a golden evaluation index of arterial stiffness suggested by ESH/ESC [2]. A recent study showed that the level of CFPWV was higher in DM subjects with higher $\mathrm{HbAlc}$ [3]. A recent review showed that PWV was a marker of cognitive impairment in the elderly subjects [4]. Our previous studies also showed that CFPWV was positively correlated with pulse pressure and it was increased in hypertension patients with left ventricular hypertrophy $[5,6]$. And CFPWV was significantly higher in healthy subjects with positive family history of hypertension [7].

As we know, hyperglycemia and hyperlipidemia are traditional risk factors leading to the development of vascular-related diseases. However, the incidence of vascularrelated diseases was still increasing after glucose and lipid control. So, there might be other risk factors involving the mechanism of vascular-related diseases or inappropriate therapeutic targets during treatment. Recent studies showed that plasma biomarkers such as homocysteine (Hcy), 
$\mathrm{N}$-terminal pro-brain natriuretic peptide (NT-proBNP), and urine albumin (microalbumin) (UAE) have involved the pathophysiological development of arteriosclerosis and could be predictors of future cardiovascular events. For example, chronic hyperhomocysteinemia contributed to coronary artery disease by inhibiting dysfunction of the coronary artery endothelium [8]. And our previous study showed that arterial stiffness was positively correlated with NT-proBNP in hypertension subjects with CHD [9]. UAE could be a marker of microvascular lesion, and it increased significantly with the severity of hypertension [10]. The levels of biomarkers could reflect the severity of vascular-related diseases. In addition, biomarker research could provide some information for the mechanism of vascular-related diseases and therapy target.

As shown above, CHD, hypertension, and DM are the most serious vascular-related diseases threatening the human's life. However, many patients were not only suffering one kind of vascular-related diseases but also suffering some of them, such as one patient might suffer hypertension, DM, and CHD simultaneously in our routine clinical examination. And this is the real world about patients. The levels or relationship between CFPWV and biomarkers could be different in patients with one or more vascularrelated diseases. So, our present was carried out to investigate the relationship between CFPWV and biomarkers in these subjects, to provide some information for the mechanism and prevention of arteriosclerosis.

\section{Materials and Methods}

2.1. Subjects. This was a retrospective study. 656 subjects $(\mathrm{M} / \mathrm{F}, 272 / 384)$ from the Department of Vascular Medicine from January 2012 to December 2013 were enrolled into our study. Subjects with stroke, arteriosclerosis obliterans, heart failure, renal function impairment, liver function impairment, systemic inflammatory diseases, infectious disease, or cancer were excluded. 186 subjects were outpatient for health examination population without the diseases of hypertension, $\mathrm{CHD}$, and $\mathrm{DM}$. The rest of 470 subjects were inpatient. And of these 470 subjects, there were 377 patients with hypertension, 231 with CHD, and 154 with DM.

We divided our study group into four groups according to the numbers of suffered diseases: healthy subjects $(N=186$, without the diseases of hypertension, CHD, and $\mathrm{DM})$, group $1(N=237$, with one of the diseases of hypertension, CHD, and DM), group $2(N=174$, with two of the diseases of hypertension, $\mathrm{CHD}$, and $\mathrm{DM}$ ), and group 3 $(N=59$, with all diseases of hypertension, CHD, and DM).

Hypertension was defined as blood pressure measurement $\geq 140 / 90 \mathrm{mmHg}$ in three occasions at rest or subjects with known cases of diagnosed hypertension before and taking antihypertensive drugs at present. CHD was defined as the narrowing $(>75 \%)$ or blockage of coronary artery diagnosed by angiography. DM was defined according to the $75 \mathrm{~g}$ oral glucose tolerance test (OGTT). All participants gave their written informed consent. This study was approved by the Ethics Committee of Peking University Shougang Hospital, China.
2.2. The Assessment of ABI. ABI was recorded using a VaseraVS-1000 vascular screening system (Fukuda Denshi, Tokyo, Japan) with the participant resting in a supine position. ECG electrodes were placed on both wrists, a microphone for detecting heart sounds was placed on the sternum, and cuffs were wrapped around both the arms and ankles. After automatic measurements, obtained data were analyzed by software, and the value of ABI was obtained automatically.

2.3. Pulse Wave Velocity Measurement. Arterial stiffness was evaluated by measuring automatic PWV using the Complior apparatus. The basic principle of PWV assessment is that pressure pulse generated by ventricular ejection is propagated along the arterial system at a speed determined by elasticity of the arterial wall. Knowing the distance and pulse transit time, the velocity can be calculated. Patients were placed in recumbent position and, after a 10-minute rest, underwent PWV measurement, and CFPWV was obtained automatically [11].

2.4. Laboratory Measurements. Blood samples were drawn from an antecubital vein in the morning after overnight fasting and collected into vacuum tubes containing EDTA for the measurement of plasma lipid and lipoprotein levels. Fasting plasma glucose (FPG), total cholesterol (TC), highdensity lipoprotein cholesterol (HDL-C), triglyceride (TG), and homocysteine levels were analyzed by colorimetric enzymatic assays with the use of an autoanalyzer (HITACHI-7170, Hitachi, Tokyo, Japan) at the central chemistry laboratory of the Peking University Shougang Hospital. Low-density lipoprotein cholesterol (LDL-C) levels were calculated. Urine albumin (microalbumin) excretion (UAE) was determined by the 12-hour overnight urine samples calculated from the urine albumin concentration, urine volume, and collection time at the central chemistry laboratory of the Peking University Shougang Hospital. The level of NT-proBNP was measured using the method of Biodirectional lateral flow immunoassay (ReLIA) according to the procedure.

2.5. Statistical Analysis. SPSS was used in the statistical analysis. The one-sample Kolmogorov-Smirnov test was used to test the normal distribution. The levels of NTproBNP and UAE were not in normal distribution, so we made log transformation of NT-proBNP and UAE. The differences between groups were analyzed by one-way ANOVA and least-significant difference (LSD). Proportions were analyzed by $\chi^{2}$-test. Correlation coefficient was done to find linear relation between different variables using Pearson correlation coefficient. Partial correlation analysis was also used in the present study. Multivariate linear regressions (stepwise) were used to estimate the coefficients of the linear equation, involving independent variables including age, BMI, SBP, DBP, pulse pressure, FPG, UA, creatinine, TC, HDL-C, LDL-C, TG, Log NT-proBNP, Hcy, and Log UAE that affected the value of CFPWV. And the $F$ value of 
multivariate linear regressions and $\mathrm{Fa}(k, n-k-1)$ value were also calculated to judge whether it was valid or not. Values were shown as mean \pm SD unless stand otherwise. In addition, the one-sample Kolmogorov-Smirnov test showed that Log UAE was not in normal distribution in group 2 $(p=0.020)$, with normal distribution in the healthy group, group 1, and group 3 (all $p>0.05$ ). In addition, Log BNP was in normal distribution in all subgroups. And Log UAE and Log NT-proBNP were not in normal distribution in the CHD group and non-CHD group. So, Me (Q1-Q3) for Log UAE and Log NT-proBNP was presented. $p<0.05$ (2-tailed) was considered statistically significant.

\section{Results}

3.1. Clinical Characteristics of the Study Participants. The baseline clinical characteristics of study participants are shown in Table 1. As shown in Table 1, 237 patients had only one of these three vascular-related diseases, 174 patients had two of these three vascular-related diseases, 59 patients had all of these three diseases, and 186 subjects with none of these three vascular-related diseases.

Our results showed that with the increasing numbers of suffered vascular-related diseases, the level of CFPWV was increasing. Similar results were also found in the parameters of biomarkers of Hcy, Log NT-ProBNP, and Log UAE. In addition, the levels of ABI and HDL-C were decreasing with the increasing numbers of suffered diseases.

In addition, CHD was accompanied with structural atherosclerotic change resulted from hypertension and DM. And we divided these subjects into two groups according to with or without CHD. As shown in Table 2, the levels of CFWV, uric acid, Hcy, Log NT-ProBNP, and Log UAE were significantly higher in the CHD group. The level of LDL-C was lower in the CHD group, according to higher usage of lipid-lowering agents.

\subsection{Pearson Correlations between CFPWV and Metabolic} Markers. Next, we investigated the Pearson correlations between biomarkers and the vascular function index in the entire group and in subgroups. Our results showed that Log NT-proBNP was positively correlated with CFPWV in the entire study group $(r=0.119, p<0.05)$ and nonhealthy group ( $r=0.164, p<0.05)$. There was negative correlation between Log NT-proBNP and RABI and LABI in the entire study group $(r=-0.171$ and -0.145 , both $p<0.05$, respectively) and nonhealthy group ( $r=-0.197$ and -0.206 , both $p<0.05$, respectively). There was positive correlation between Hcy and CFPWV in the entire study group $(r=0.109$, $p<0.05)$ and nonhealthy group $(r=0.093, p=0.050)$. In addition, our present study showed that there was positive correlation between Log UAE and CFPWV in the entire study group $(r=0.196, p<0.05)$ and nonhealthy group $(r=0.209, p<0.050)$.

As shown above, there was significant difference about age, drug usage, BMI, blood pressure, FPG, uric acid, and lipids between these groups. So, we made partial correlation analysis next. And our present result showed that there was positive correlation between Log UAE and CFPWV in the entire study group $(r=0.105, p=0.050)$ after adjusting for age, BMI, FPG, blood pressure, uric acid, and lipids without significant difference between Log NT-proBNP, Hcy, and CFPWV after adjusting.

As shown in Table 3, subgroup Pearson correlation analysis showed that there was positive correlation between CFPWV and age, SBP, and pulse pressure in all subgroups. And significant negative correlation between CFPWV and RABI $(r=-0.315, p=0.015)$ was found in group 3 , with negative correlation tendency between CFPWV and LABI $(r=-0.239, p=0.069)$.

3.3. Multivariate Regression Analysis. Multivariate linear regressions (stepwise) were used to estimate the coefficients of the linear equation, involving independent variables including age, BMI, SBP, DBP, pulse pressure, FPG, UA, creatinine, TC, HDL-C, LDL-C, TG, Log NT-proBNP, Hcy, and Log UAE that affected the value of CFPWV. The $F$ value of multivariate linear regressions was $36.881(p<0.001)$, and the $\mathrm{Fa}(0.05,15,656)$ value was 1.68 , so it was valid. Our results showed that pulse pressure, age, FPG, DBP, BMI, and Log UAE were independent associating factors of CFPWV in all subjects $(\beta=0.334, p<0.001 ; \beta=0.333, p<0.001 ; \beta=0.126$, $p=0.004 ; \beta=0.137, p=0.003 ; \beta=-0.142, p=0.002$; and $\beta=0.098, p=0.031$, respectively, Tables 4 and 5).

\section{Discussion}

Our present study showed that CFPWV was significantly higher in subjects with hypertension, CHD, and DM, with decreasing level of ABI. And there was correlation between CFPWV and biomarkers such as Log NT-proBNP, Hcy, and Log UAE.

CHD, hypertension, and DM are the most serious vascular-related diseases with similar pathophysiological changes such as arteriosclerosis. Arteriosclerosis could be evaluated by arterial stiffness, which is measured by CFPWV suggested by ESH/ESC [2]. A previous study showed that aortic PWV was an independent predictor of coronary heart disease and aortic PWV also provided additional predictive value above cardiovascular risk factors [12]. A prospective study showed that carotid and femoral stiffness indices were independently associated with incident cardiovascular events and all-cause mortality [13]. Mulders et al. [14] found that patients with premature $\mathrm{CHD}$ and their first-degree relatives had higher PWV compared with controls, independent of other risk factors, and Kingwell et al. reported that large artery stiffness was a major determinant for myocardial ischemic threshold in patients with coronary artery disease [15]. A recent study showed that aortic stiffness was an independent predictor of incident mild cognitive impairment and a potentially modifiable risk factor for clinical cognitive impairment and dementia [16]. These studies confirmed the role of PWV in the prediction of vascular-related events.

However, many patients were not suffering only one kind of vascular-related disease but also suffering some of 
TABLE 1: Clinical characteristics in different groups.

\begin{tabular}{|c|c|c|c|c|c|}
\hline Characteristics & Healthy $(N=186)$ & Group $1(N=237)$ & Group $2(N=174)$ & Group $3(N=59)$ & $p$ value \\
\hline Age (year) & $56.0 \pm 10.4$ & $60.0 \pm 10.0^{*}$ & $65.0 \pm 11.5^{* \#}$ & $67.2 \pm 11.7^{* \#}$ & $<0.001$ \\
\hline Male/female & $74 / 112$ & $97 / 140$ & $78 / 96$ & $23 / 36$ & 0.752 \\
\hline CCB (\%) & 0 & $18.1^{*}$ & $29.3^{* \#}$ & $49.2^{* \#} \%$ & $<0.001$ \\
\hline ACEI/ARB (\%) & 0 & $17.3^{*}$ & $28.7^{* \#}$ & $44.0^{* \#} \%$ & $<0.001$ \\
\hline B-blocker (\%) & 0 & $13.5^{*}$ & $31.0^{* \#}$ & $33.9^{* \#}$ & $<0.001$ \\
\hline Antidiabetic agent (\%) & 0 & $4.6^{*}$ & $21.2^{* \#}$ & $72.9^{* \#} \%$ & $<0.001$ \\
\hline Lipid-lowering & & & & & \\
\hline agent $(\%)$ & 0 & $27.4^{*}$ & $41.4^{* \#}$ & $54.2^{* \#} \%$ & $<0.001$ \\
\hline Smoking (\%) & 24.7 & 27.8 & 32.7 & 27.1 & 0.344 \\
\hline BMI $\left(\mathrm{kg} / \mathrm{m}^{2}\right)$ & $24.38 \pm 3.46$ & $25.65 \pm 3.43^{*}$ & $26.07 \pm 3.40^{*}$ & $26.09 \pm 3.66^{*}$ & $<0.001$ \\
\hline CFPWV $(\mathrm{m} / \mathrm{s})$ & $10.11 \pm 2.28$ & $10.70 \pm 2.12^{*}$ & $11.92 \pm 2.55^{* \#}$ & $12.71 \pm 2.38^{* \#} \%$ & $<0.001$ \\
\hline RABI & $1.130 \pm 0.10$ & $1.125 \pm 0.08$ & $1.098 \pm 0.11^{* \#}$ & $1.058 \pm 0.12^{* \#} \%$ & $<0.001$ \\
\hline LABI & $1.116 \pm 0.12$ & $1.122 \pm 0.09$ & $1.095 \pm 0.11^{\#}$ & $1.083 \pm 0.15^{* \#}$ & 0.019 \\
\hline SBP (mmHg) & $128.8 \pm 16.2$ & $139.0 \pm 19.4^{*}$ & $145.0 \pm 20.3^{*}$ & $145.0 \pm 20.0^{* \#}$ & $<0.001$ \\
\hline $\mathrm{DBP}(\mathrm{mmHg})$ & $80.7 \pm 9.5$ & $84.9 \pm 12.0^{*}$ & $86.3 \pm 11.2^{*}$ & $83.6 \pm 11.3$ & $<0.001$ \\
\hline Pulse pressure $(\mathrm{mmHg})$ & $48.1 \pm 10.6$ & $54.0 \pm 13.5^{*}$ & $58.7 \pm 16.0^{* \#}$ & $61.4 \pm 14.6^{* \#}$ & $<0.001$ \\
\hline FPG $(\mathrm{mmol} / \mathrm{L})$ & $5.50 \pm 0.85$ & $5.69 \pm 1.21$ & $6.31 \pm 1.92^{* \#}$ & $7.44 \pm 2.53^{* \#} \%$ & $<0.001$ \\
\hline $\mathrm{UA}(\mu \mathrm{mol} / \mathrm{L})$ & $298.2 \pm 71.8$ & $310.9 \pm 80.7$ & $321.9 \pm 79.3^{*}$ & $314.4 \pm 91.7$ & 0.043 \\
\hline Creatinine $(\mu \mathrm{mol} / \mathrm{L})$ & $63.2 \pm 13.5$ & $66.5 \pm 22.0$ & $68.4 \pm 19.7$ & $68.3 \pm 19.8$ & 0.055 \\
\hline $\mathrm{TC}(\mathrm{mmol} / \mathrm{L})$ & $5.18 \pm 1.10$ & $4.95 \pm 1.11$ & $4.44 \pm 1.15^{* \#}$ & $4.58 \pm 1.27^{* \#}$ & $<0.001$ \\
\hline HDL-C (mmol/L) & $1.33 \pm 0.35$ & $1.25 \pm 0.31^{*}$ & $1.14 \pm 0.27^{* \#}$ & $1.11 \pm 0.27^{* \#}$ & $<0.001$ \\
\hline LDL-C (mmol/L) & $3.25 \pm 0.89$ & $3.05 \pm 0.89$ & $2.59 \pm 0.86^{* \#}$ & $2.72 \pm 0.84^{* \#}$ & $<0.001$ \\
\hline TG (mmol/L) & $1.72 \pm 1.29$ & $1.84 \pm 1.56$ & $1.77 \pm 1.80$ & $1.96 \pm 2.32$ & 0.751 \\
\hline $\mathrm{HCY}(\mu \mathrm{mol} / \mathrm{L})$ & $11.57 \pm 5.90$ & $13.82 \pm 8.21^{*}$ & $15.27 \pm 7.21^{*}$ & $15.87 \pm 8.08^{*}$ & $<0.001$ \\
\hline Log NT-proBNP & $1.84-2.27$ & $1.89-2.33$ & $2.04-2.55^{* \#}$ & $1.92-2.57^{* \#}$ & $<0.001$ \\
\hline Log UAE & $0.36-0.76$ & $0.40-0.93$ & $0.54-1.03^{* \#}$ & $0.57-1.49^{* \#}$ & $<0.001$ \\
\hline
\end{tabular}

$p$ values were calculated using analysis of one-way ANOVA. Least-significant difference (LSD) was used to do comparison analysis between groups, marked with $*$, \#, and $\%$. ${ }^{*}$ vs healthy, $p<0.05 ;{ }^{\#}$ vs group $1, p<0.05 ; \%$ vs group $2, p<0.05$; group 1 , with one of the diseases of hypertension, CHD, and DM; group 2 , with two of the diseases of hypertension, CHD, and DM; group 3: with all the diseases of hypertension, CHD, and DM; CHD, coronary heart disease; DM, diabetes mellitus; BMI, body mass index; CFPWV, carotid-femoral pulse wave velocity; RABI, right ankle brachial index; LABI, left ankle brachial index; SBP, systolic blood pressure; DBP, diastolic blood pressure; FPG, fasting plasma glucose; UA, uric acid; TC, cholesterol; LDL-C, low-density lipoprotein cholesterol; HDL-C, high-density lipoprotein cholesterol; TG, triglycerides; HCY, homocysteine; UAE, urine albumin (microalbumin) excretion; CCB, calcium channel blocker; ACEI, angiotensin-converting enzyme inhibitors; ARB, angiotensin receptor blocker.

them, such as one patient might be found to have hypertension, DM, and CHD simultaneously in our routine clinical examination. And this is the real world about patients. And our present study showed that CFPWV was increasing with the increasing number of suffered diseases, indicating that multiple diseases could worsen arterial stiffness. So, arterial stiffness should be more emphasized in order to reduce vascular-related events in future clinical therapy.

Biomarkers of vascular-related diseases such as homocysteine (Hcy), N-terminal pro-brain natriuretic peptide (NT-proBNP), and urine albumin (microalbumin) (UAE) have involved the pathophysiological development of arteriosclerosis. Hyperhomocysteinemia (HHcy) has been considered as an independent risk factor for atherosclerosis [17]. And a recent study showed that plasma total homocysteine was an independent risk factor for stroke [18]. Our present study showed that Hcy was increasing the increasing number of suffered diseases, and positive correlation was found between Hcy and CFPWV. NT-proBNP is a controregulatory hormone associated with cardiac remodeling such as left ventricular hypertrophy and systolic/diastolic dysfunction. In addition to the role of NT-proBNP in the disease of heart failure, a recent study showed that NTproBNP is a more significant prognostic factor for cardiovascular mortality [19]. In addition, our previous study also showed the similar result about the relationship between arterial stiffness and NT-proBNP in hypertension subjects with coronary artery disease [9].

Microalbuminuria was an early indicator of impaired renal function caused by hypertension, and the UAE increased significantly with the severity of hypertension [20]. A recent study showed that low levels of albuminuria were associated with increased risk of incident hypertension and CHD mortality at follow-up [21]. And microalbuminuria was significantly correlated to diabetes duration [22]. A recent review showed that microalbuminuria might not simply be regarded as a risk predictor but become itself an independent target for treatment of cardiovascular events $[23,24]$. Metabolic syndrome and all its components were associated with the presence of microalbuminuria in a health checkup population in China [25]. Our present study showed that level of Log UAE was increasing with the number of suffering diseases increasing, and there was positive correlation between Log UAE and CFPWV. In addition, multivariate linear regression analysis showed that Log UAE was an independent associating factor of CFPWV. In addition, DBP and FPG were independent associating factors of CFPWV, and Log UAE was an indicator of vascular-related diseases complications of renal dysfunction, 
TABLE 2: Clinical characteristics in different groups.

\begin{tabular}{|c|c|c|c|}
\hline Characteristics & Non-CHD group $(N=425)$ & CHD group $(N=231)$ & $p$ \\
\hline Age (year) & $58.3 \pm 10.7$ & $65.3 \pm 11.3$ & $<0.001$ \\
\hline Male/female & $168 / 257$ & $104 / 127$ & 0.173 \\
\hline Hypertension (\%) & 49.8 & 73.5 & $<0.001$ \\
\hline $\mathrm{DM}(\%)$ & 18.6 & 32.5 & $<0.001$ \\
\hline CCB $(\%)$ & 13.2 & 29.4 & $<0.001$ \\
\hline ACEI/ARB (\%) & 12.7 & 28.1 & $<0.001$ \\
\hline B-blocker (\%) & 7.8 & 43.6 & $<0.001$ \\
\hline Antidiabetic agent (\%) & 9.2 & 22.9 & $<0.001$ \\
\hline $\begin{array}{l}\text { Lipid-lowering } \\
\text { Agent (\%) }\end{array}$ & 23.1 & 46.3 & $<0.001$ \\
\hline Smoking (\%) & 32.2 & 36.4 & 0.285 \\
\hline BMI $\left(\mathrm{kg} / \mathrm{m}^{2}\right)$ & $25.18 \pm 3.46$ & $25.94 \pm 3.57$ & 0.008 \\
\hline $\operatorname{CFPWV}(\mathrm{m} / \mathrm{s})$ & $10.72 \pm 2.37$ & $11.63 \pm 2.52$ & $<0.001$ \\
\hline RABI & $1.125 \pm 0.099$ & $1.091 \pm 0.108$ & $<0.001$ \\
\hline LABI & $1.116 \pm 0.107$ & $1.099 \pm 0.119$ & 0.076 \\
\hline SBP (mmHg) & $136.6 \pm 19.3$ & $141.2 \pm 20.0$ & 0.004 \\
\hline DBP $(\mathrm{mmHg})$ & $84.1 \pm 11.0$ & $83.6 \pm 11.8$ & 0.574 \\
\hline Pulse pressure (mmHg) & $52.4 \pm 13.4$ & $57.6 \pm 15.2$ & $<0.001$ \\
\hline FPG $(\mathrm{mmol} / \mathrm{L})$ & $5.82 \pm 1.32$ & $6.21 \pm 2.02$ & 0.010 \\
\hline $\mathrm{UA}(\mu \mathrm{mol} / \mathrm{L})$ & $304.6 \pm 76.5$ & $321.5 \pm 83.3$ & 0.010 \\
\hline Creatinine $(\mu \mathrm{mol} / \mathrm{L})$ & $65.3 \pm 19.3$ & $67.9 \pm 18.9$ & 0.089 \\
\hline $\mathrm{TC}(\mathrm{mmol} / \mathrm{L})$ & $5.02 \pm 1.17$ & $4.52 \pm 1.10$ & $<0.001$ \\
\hline HDL-C (mmol/L) & $1.28 \pm 0.33$ & $1.14 \pm 0.27$ & $<0.001$ \\
\hline $\mathrm{LDL}-\mathrm{C}(\mathrm{mmol} / \mathrm{L})$ & $3.11 \pm 0.92$ & $2.65 \pm 0.82$ & $<0.001$ \\
\hline TG $(\mathrm{mmol} / \mathrm{L})$ & $1.77 \pm 1.40$ & $1.86 \pm 2.05$ & 0.496 \\
\hline $\mathrm{HCY}(\mu \mathrm{mol} / \mathrm{L})$ & $12.73 \pm 7.18$ & $15.58 \pm 7.68$ & $<0.001$ \\
\hline Log NT-proBNP & $1.89-2.31$ & $1.99-.57$ & $<0.001$ \\
\hline Log UAE & $0.40-0.91$ & $0.47-0.99$ & 0.028 \\
\hline
\end{tabular}

BMI, body mass index; CFPWV, carotid-femoral pulse wave velocity; RABI, right ankle brachial index; LABI, left ankle brachial index; SBP, systolic blood pressure; DBP, diastolic blood pressure; FPG, fasting plasma glucose; UA, uric acid; TC, cholesterol; LDL-C, low-density lipoprotein cholesterol; HDL-C, high-density lipoprotein cholesterol; TG, triglycerides; HCY, homocysteine; UAE, urine albumin (microalbumin) excretion; CCB, calcium channel blocker; ACEI, angiotensin-converting enzyme inhibitors; ARB, angiotensin receptor blocker.

TABle 3: Pearson correlations between CFPWV and metabolic markers in each subgroup.

\begin{tabular}{|c|c|c|c|c|c|c|c|c|}
\hline \multirow{2}{*}{ Characteristics } & \multicolumn{2}{|c|}{ Healthy $(N=186)$} & \multicolumn{2}{|c|}{ Group $1(N=237)$} & \multicolumn{2}{|c|}{ Group $2(N=174)$} & \multicolumn{2}{|c|}{ Group $3(N=59)$} \\
\hline & $r$ & $p$ & $r$ & $p$ & $r$ & $p$ & $r$ & $p$ \\
\hline Age (year) & 0.451 & $<0.001$ & 0.327 & $<0.001$ & 0.457 & $<0.001$ & 0.392 & 0.002 \\
\hline BMI $\left(\mathrm{kg} / \mathrm{m}^{2}\right)$ & -0.114 & 0.126 & -0.059 & 0.370 & -0.033 & 0.668 & -0.228 & 0.083 \\
\hline RABI & -0.006 & 0.934 & -0.063 & 0.337 & -0.250 & 0.001 & -0.315 & 0.015 \\
\hline LABI & 0.069 & 0.349 & -0.152 & 0.019 & -0.386 & $<0.001$ & -0.239 & 0.069 \\
\hline $\mathrm{SBP}(\mathrm{mmHg})$ & 0.357 & $<0.001$ & 0.302 & $<0.001$ & 0.476 & $<0.001$ & 0.480 & $<0.001$ \\
\hline DBP (mmHg) & 0.122 & 0.098 & 0.166 & 0.011 & 0.224 & 0.003 & 0.003 & 0.983 \\
\hline Pulse pressure (mmHg) & 0.439 & $<0.001$ & 0.285 & $<0.001$ & 0.448 & $<0.001$ & 0.574 & $<0.001$ \\
\hline FPG $(\mathrm{mmol} / \mathrm{L})$ & 0.091 & 0.219 & 0.068 & 0.295 & 0.071 & 0.353 & -0.031 & 0.818 \\
\hline $\mathrm{UA}(\mu \mathrm{mol} / \mathrm{L})$ & 0.017 & 0.816 & 0.286 & $<0.001$ & -0.156 & 0.041 & -0.061 & 0.646 \\
\hline Creatinine $(\mu \mathrm{mol} / \mathrm{L})$ & 0.171 & 0.021 & 0.165 & 0.011 & 0.190 & 0.012 & 0.103 & 0.440 \\
\hline $\mathrm{TC}(\mathrm{mmol} / \mathrm{L})$ & 0.077 & 0.296 & -0.042 & 0.527 & -0.066 & 0.388 & 0.048 & 0.719 \\
\hline HDL-C (mmol/L) & 0.032 & 0.668 & -0.077 & 0.240 & 0.052 & 0.496 & 0.239 & 0.071 \\
\hline $\mathrm{LDL}-\mathrm{C}(\mathrm{mmol} / \mathrm{L})$ & -0.029 & 0.696 & -0.079 & 0.231 & -0.038 & 0.621 & -0.020 & 0.880 \\
\hline TG $(\mathrm{mmol} / \mathrm{L})$ & 0.074 & 0.318 & 0.095 & 0.147 & -0.187 & 0.014 & 0.001 & 0.994 \\
\hline $\mathrm{HCY}(\mu \mathrm{mol} / \mathrm{L})$ & -0.034 & 0.650 & 0.143 & 0.033 & 0.045 & 0.571 & -0.172 & 0.205 \\
\hline Log NT-proBNP & 0.155 & 0.057 & -0.004 & 0.959 & 0.224 & 0.011 & 0.193 & 0.175 \\
\hline Log UAE & 0.068 & 0.376 & -0.101 & 0.139 & 0.102 & 0.210 & -0.181 & 0.181 \\
\hline
\end{tabular}

Group 1, with one of the diseases of hypertension, CHD, and DM; group 2, with two of the diseases of hypertension, CHD, and DM; group 3: with all the diseases of hypertension, CHD, and DM; CHD, coronary heart disease; DM, diabetes mellitus; BMI, body mass index; CFPWV, carotid-femoral pulse wave velocity; RABI, right ankle brachial index; LABI, left ankle brachial index; SBP, systolic blood pressure; DBP, diastolic blood pressure; FPG, fasting plasma glucose; UA, uric acid; TC, cholesterol; LDL-C, low-density lipoprotein cholesterol; HDL-C, high-density lipoprotein cholesterol; TG, triglycerides; HCY, homocysteine; UAE, urine albumin (microalbumin) excretion. 
TABLE 4: Multiple linear regression analysis for the relationship between CFPWV and study variables among the entire study group, independent associating factors of CFPWV in all subjects.

\begin{tabular}{lccccc}
\hline & Unstandardized $\beta$ & $95 \%$ CI for $\beta$ & Std. error & Standardized $\beta$ & $t$ \\
\hline Constant & 1.786 & $(-0.681,4.253)$ & 1.254 & - & 1.424 \\
Pulse pressure & 0.057 & $(0.041,0.074)$ & 0.008 & 0.334 & 6.930 \\
Age & 0.074 & $(0.053,0.094)$ & 0.010 & 0.333 & 7.107 \\
FPG & 0.188 & $(0.060,0.315)$ & 0.065 & 0.126 & $<0.001$ \\
DBP & 0.032 & $(0.011,0.053)$ & 0.011 & 0.137 & 2.899 \\
BMI & -0.104 & $(-0.168,-0.039)$ & 0.033 & -0.142 & 0.004 \\
Log UAE & 0.491 & $(0.046,0.936)$ & 0.226 & 0.098 & -3.152 \\
\hline
\end{tabular}

CFPWV, carotid-femoral pulse wave velocity; BMI, body mass index; DBP, diastolic blood pressure; FPG, fasting plasma glucose; UAE, urine albumin (microalbumin) excretion; CI, confidence interval; SBP, systolic blood pressure; UA, uric acid; TC, cholesterol; LDL-C, low-density lipoprotein cholesterol; HDL-C, high-density lipoprotein cholesterol; TG, triglycerides; HCY, homocysteine.

this might indicate the relationship between early renal dysfunction and CFPWV. Andfurther study should be done in future. In addition, similar to the result of the present study, Kong et al. found that arterial stiffness increases the risk of proteinuria in a Chinese population-based cohort [26].

Our present study showed that arterial stiffness was increasing with the number of suffered vascular-related diseases increasing, with the levels of some biomarkers increasing. As shown above, arterial stiffness and biomarkers could both be the predictors of future cardiovascular events. So, the predictive value might be improved by combination of arterial stiffness and biomarkers together. A recent study showed that aortic PWV improves risk prediction when added to standard risk factors [27]. Blankenberg et al. showed that combing traditional risk factors with NT-proBNP can provide the best clinical prediction of recurrent cardiovascular events in the secondary-prevention population [28]. In addition, the MORGAM study confirmed that adding N-terminal pro-brain natriuretic peptide, C-reactive protein, and sensitive troponin I to a conventional risk model can improve 10-year risk estimation for cardiovascular events [29]. So, we might collect some arterial function indexes and some biomarkers together, called early vascular lesion detection technology, to provide some information for the complete and accurate evaluation of one patient. We established the early vascular lesion detection system and published the first guideline in 2005 [30], the Chinese Guideline for Early Vascular Disease Detection (first report). This guideline was renewed in 2011 as the Chinese Guideline for Early Vascular Disease Detection (2011, second report) [31].

A major limitation of our study was its cross-sectional design; another limitation was that the subjects' numbers of each group were not balanced. In addition, some patients with CHD or hyperlipids had statins when enrolled into our present study, so the level of LDL-C was decreasing in subjects with two or more kinds of diseases. In addition, our previous research [32] about relationship between the cardio-ankle vascular index (CAVI) and biomarkers in vascular-related diseases showed that age, BMI, Log UAE, and DBP were independent associating factors of the cardioankle vascular index in all subjects. Both PWV and CAVI are the evaluation index of arterial stiffness with different points
TABLE 5: Multiple linear regression analysis for the relationship between CFPWV and study variables among the entire study group, excluded variables by multiple linear regression analysis in all subjects.

\begin{tabular}{lccc}
\hline Characteristics & Beta in & $t$ & $p$ value \\
\hline SBP $(\mathrm{mmHg})$ & -0.473 & -0.328 & 0.743 \\
UA $(\mu \mathrm{mol} / \mathrm{L})$ & 0.072 & 1.665 & 0.097 \\
TC $(\mathrm{mmol} / \mathrm{L})$ & -0.044 & -1.050 & 0.295 \\
HDL-C $(\mathrm{mmol} / \mathrm{L})$ & -0.060 & -1.344 & 0.180 \\
LDL-C $(\mathrm{mmol} / \mathrm{L})$ & -0.082 & -1.950 & 0.052 \\
TG $(\mathrm{mmol} / \mathrm{L})$ & 0.056 & 1.283 & 0.200 \\
HCY $(\mu \mathrm{mol} / \mathrm{L})$ & -0.006 & -0.136 & 0.892 \\
Log NT-proBNP & -0.044 & -0.957 & 0.339 \\
Creatinine $(\mu \mathrm{mol} / \mathrm{L})$ & 0.058 & 1.384 & 0.167 \\
\hline
\end{tabular}

CFPWV, carotid-femoral pulse wave velocity; BMI, body mass index; DBP, diastolic blood pressure; FPG, fasting plasma glucose; UAE, urine albumin (microalbumin) excretion; CI, confidence interval; SBP, systolic blood pressure; UA, uric acid; TC, cholesterol; LDL-C, low-density lipoprotein cholesterol; HDL-C, high-density lipoprotein cholesterol; TG, triglycerides; HCY, homocysteine.

of focus [33]. However, our present study might provide some information about relationship between arterial stiffness and biomarkers. So, more studies and analysis should be investigated in future (Table 5).

In conclusion, our present study showed that CFPWV was significantly higher in subjects with hypertension, $\mathrm{CHD}$, and DM with decreasing level of ABI. And there was correlation between CFPWV and biomarkers such as NTproBNP, Hcy, and UAE. And a vascular lesion evaluation system including arterial function and biomarkers could be founded in future.

\section{Data Availability}

No additional data are available.

\section{Ethical Approval}

This study was approved by the Ethics Committee of the Peking University Shougang Hospital, China.

\section{Conflicts of Interest}

The authors declare that they have no conflicts of interest. 


\section{Authors' Contributions}

Jinbo Liu and Kuanting Wang equally contributed to this work.

\section{Acknowledgments}

This work was supported by grants from the hospital fund of the Peking University Shougang Hospital to Hongyu Wang (nos. SGYYZ201610 and 2017-Hospital-Clinical-01) and Jinbo Liu (SGYYQ201605), and National Key Research and Development Plan (2017YFC0113005).

\section{References}

[1] G. F. Mitchell, "Aortic stiffness, pressure and flow pulsatility, and target organ damage," Journal of Applied Physiology, vol. 125, no. 6, pp. 1871-1880, 2018.

[2] P. K. Whelton, R. M. Carey, W. S. Aronow et al., "2017 ACC/ AHA/AAPA/ABC/ACPM/AGS/APhA/ASH/ASPC/NMA/ PCNA guideline for the prevention, detection, evaluation, and management of high blood pressure in adults: a report of the American college of cardiology/American heart association task force on clinical practice guidelines," Hypertension, vol. 72, no. 3, pp. e13-e115, 2018.

[3] M. T. Ferreira, N. C. Leite, C. R. L. Cardoso, and G. F. Salles, "Correlates of aortic stiffness progression in patients with type 2 diabetes: importance of glycemic control," Diabetes Care, vol. 38, no. 5, pp. 897-904, 2015.

[4] A. Scuteri and H. Wang, "Pulse wave velocity as a marker of cognitive impairment in the elderly," Journal of Alzheimer's Disease, vol. 42, no. s4, pp. S401-S410, 2014.

[5] Y. Ni, H. Wang, D. Hu, and W. Zhang, "The relationship between pulse wave velocity and pulse pressure in Chinese patients with essential hypertension," Hypertension Research, vol. 26, no. 11, pp. 871-874, 2003.

[6] H. Wang, W. Zhang, and L. Gong, "Study of relationship between large artery distensibility and left ventricular hypertrophy in patients with essential hypertension," Chinese Journal of Cardiology, vol. 28, pp. 177-180, 2000.

[7] J. Liu, H. Wang, H. Zhao, H. Liu, L. Li, and Y. Zhou, "Arterial stiffness is increased in healthy subjects with a positive family history of hypertension," Clinical and Experimental Hypertension, vol. 37, no. 8, pp. 622-626, 2015.

[8] L. He, H. Zeng, F. Li et al., "Homocysteine impairs coronary artery endothelial function by inhibiting tetrahydrobiopterin in patients with hyperhomocysteinemia," American Journal of Physiology-Endocrinology and Metabolism, vol. 299, no. 6, pp. E1061-E1065, 2010.

[9] H. Wang, J. Liu, H. Zhao et al., "Relationship between cardioankle vascular index and n-terminal pro-brain natriuretic peptide in hypertension and coronary heart disease subjects," Journal of the American Society of Hypertension, vol. 8, no. 9, pp. 637-643, 2014.

[10] L. Yue, Z. Fan, L. Sun, W. Feng, and J. Li, "Prevalence of essential hypertension and its complications among Chinese population at high altitude," High Altitude Medicine \& Biology, vol. 18, no. 2, pp. 140-144, 2017.

[11] R. Asmar, A. Benetos, J. Topouchian et al., "Assessment of arterial distensibility by automatic pulse wave velocity measurement," Hypertension, vol. 26, no. 3, pp. 485-490, 1995.

[12] M. A. Said, R. N. Eppinga, E. Lipsic, N. Verweij, and P. Van Der Harst, "Relationship of arterial stiffness index and pulse pressure with cardiovascular disease and mortality," Journal of the American Heart Association, vol. 7, no. 2, Article ID e007621, 2018.

[13] T. T. Van Sloten, M. T. Schram, K. Van Den Hurk et al., "Local stiffness of the carotid and femoral artery is associated with incident cardiovascular events and all-cause mortality," Journal of the American College of Cardiology, vol. 63, no. 17, pp. 1739-1747, 2014.

[14] T. A. Mulders, B. Van Den Bogaard, A. Bakker et al., "Arterial stiffness is increased in families with premature coronary artery disease," Heart, vol. 98, no. 6, pp. 490-494, 2012.

[15] B. A. Kingwell, T. K. Waddell, T. L. Medley, J. D. Cameron, and A. M. Dart, "Large artery stiffness predicts ischemic threshold in patients with coronary artery disease," Journal of the American College of Cardiology, vol. 40, no. 4, pp. 773-779, 2002.

[16] M. P. Pase, A. Beiser, J. J. Himali et al., "Aortic stiffness and the risk of incident mild cognitive impairment and dementia," Stroke, vol. 47, no. 9, pp. 2256-2261, 2016.

[17] C. Antoniades, A. S. Antonopoulos, D. Tousoulis, K. Marinou, and C. Stefanadis, "Homocysteine and coronary atherosclerosis: from folate fortification to the recent clinical trials," European Heart Journal, vol. 30, no. 30, pp. 6-15, 2009.

[18] H. Pang, Q. Fu, Q. Cao, L. Hao, and Z. Zong, "Sex differences in risk factors for stroke in patients with hypertension and hyperhomocysteinemia," Scientific Reports, vol. 9, no. 1, p. 14313, 2019.

[19] H. J. Oh, M. J. Lee, Y. E. Kwon et al., "Which biomarker is the best for predicting mortality in incident peritoneal dialysis patients: NT-ProBNP, cardiac TnT, or hsCRP?: a prospective observational study," Medicine, vol. 94, no. 44, Article ID e1636, 2015.

[20] J. T. Flynn, "Microalbuminuria in children with primary hypertension," The Journal of Clinical Hypertension, vol. 18, no. 10, pp. 962-965, 2016.

[21] K. C. Sung, S. Ryu, J. Y. Lee et al., "Urine albumin/creatinine ratio below $30 \mathrm{mg} / \mathrm{g}$ is a predictor of incident hypertension and cardiovascular mortality," Journal of the American Heart Association, vol. 13, no. 9, p. 5, 2016.

[22] D. Agnoletti, A. S. Mansour, Y. Zhang et al., "Clinical interaction between diabetes duration and aortic stiffness in type 2 diabetes mellitus," Journal of Human Hypertension, vol. 31, no. 3, pp. 189-194, 2016.

[23] F. Viazzi, F. Cappadona, and R. Pontremoli, "Microalbuminuria in primary hypertension: a guide to optimal patient management?" Journal of Nephrology, vol. 29, no. 6, pp. 747-753, 2016.

[24] P. Purohit, K. Garg, V. Singh, S. Dwivedi, and P. Sharma, "Microalbuminuria in obese young and middle aged population: a potential marker of cardiovascular risk," Indian Journal of Clinical Biochemistry, vol. 31, no. 3, pp. 349-352, 2016.

[25] Y. Y. Lee, C. K. Yang, Y. M. Weng et al., "All components of metabolic syndrome are associated with microalbuminuria in a Chinese population," PLoS One, vol. 11, no. 6, Article ID e0157303, 2016.

[26] X. Kong, X. Ma, L. Tang et al., "Arterial stiffness evaluated by carotid-femoral pulse wave velocity increases the risk of chronic kidney disease in a Chinese population-based cohort," Nephrology, vol. 22, no. 3, pp. 205-212, 2017.

[27] C. Palombo and M. Kozakova, "Arterial stiffness, atherosclerosis and cardiovascular risk: pathophysiologic mechanisms and emerging clinical indications," Vascular Pharmacology, vol. 77, pp. 1-7, 2016. 
[28] S. Blankenberg, M. J. McQueen, M. Smieja et al., "Comparative impact of multiple biomarkers and n-terminal pro-brain natriuretic peptide in the context of conventional risk factors for the prediction of recurrent cardiovascular events in the heart outcomes prevention evaluation (hope) study," Circulation, vol. 114, no. 3, pp. 201-208, 2006.

[29] S. Blankenberg, T. Zeller, O. Saarela et al., "Contribution of 30 biomarkers to 10-year cardiovascular risk estimation in 2 population cohorts," Circulation, vol. 121, no. 22, pp. $2388-2397,2010$.

[30] Chinese Medical Foundation Vascular Protection Branch, "Chinese guideline for early vascular disease detection (first report)," Medical Journal of Chinese People's Health, vol. 18, pp. 323-331, 2006.

[31] Chinese Medical Foundation Vascular Protection Branch, "Chinese guideline for early vascular disease detection (2011 second report)," Therapeutic Advances in Cardiovascular Disease, vol. 32, no. 3, pp. 318-323, 2011.

[32] J. Liu, H. Liu, H. Zhao et al., "Descriptive study of relationship between cardio-ankle vascular index and biomarkers in vascular-related diseases," Clinical and Experimental Hypertension, vol. 39, no. 5, pp. 1-5, 2017.

[33] V. V. S. Bonarjee, "Arterial stiffness: a prognostic marker in coronary heart disease. available methods and clinical application," Frontiers in Cardiovascular Medicine, vol. 5, p. 64, 2018. 